\title{
Clinical profile of individuals with bisphosphonate-related osteonecrosis of the jaw: an integrative review
}

\author{
Aloizio Premoli Maciel', Reyna Aguilar Quispe", Lázara Joyce Oliveira Martins"', Rogério Jardim Caldas"v, Paulo Sérgio da Silva Santos ${ }^{\vee}$
}

Faculdade de Odontologia da Universidade de São Paulo (FOUSP), Bauru (SP), Brazil

'DDS, MSc. Dentist and Doctoral Student, Department of Surgery, Stomatology, Pathology and Radiology, Faculdade de Odontologia da Universidade de São Paulo (FOUSP), Bauru (SP), Brazil.

(D) orcid.org/0000-0002-7062-0630

"DDS, MSc. Dentist and Doctoral Student, Department of Surgery, Stomatology, Pathology and Radiology, Faculdade de Odontologia da Universidade de São Paulo (FOUSP), Bauru (SP), Brazil.

(D) orcid.org/0000-0002-1231-5515

"'DDS, MSc. Dentist and Assistant Professor, Department of Radiology, Clinical School of Dentistry, Universidade de Rio Verde (UniRV), Rio Verde (GO), Brazil.

(D) orcid.org/0000-0003-3512-5447

"DDS, MSc, PhD. Dentist and Head, Department of Dentistry, Hospital Mário Kroeff - Hospital de Câncer, Rio de Janeiro (RJ), Brazil.

(D) orcid.org/0000-0001-6410-0551

'DDS, MSc, PhD. Dentist and Associate Professor, Department of Surgery, Stomatology, Pathology and Radiology, Faculdade de Odontologia da Universidade de São Paulo (FOUSP), Bauru, São Paulo, Brazil.

(D) orcid.org/0000-0002-0674-3759

KEY WORDS (MeSH terms):

Osteonecrosis.

Jaw.

Diphosphonates

Bisphosphonate-associated osteonecrosis of the jaw

Dental care.

Angiogenesis inhibitors.

\section{AUTHORS' KEY WORDS:}

Osteonecrosis of the jaw.

Medication-related osteonecrosis of the jaw. Neoplasm metastasis therapy.

Diagnosis and management of osteonecrosis of the jaw.

Drug-induced osteonecrosis of the jaw.

\begin{abstract}
BACKGROUND: Bisphosphonate-related osteonecrosis of the jaw (BRONJ) is still the most prevalent type of osteonecrosis with clinical relevance. In Brazil, bisphosphonate use is high but there is a lack of epidemiological studies on BRONJ.

OBJECTIVE: To determine the clinical profile of BRONJ in a Brazilian population through an integrative review.

DESIGN AND SETTING: Integrative review of BRONJ in a Brazilian population.

METHODS: Cases and clinical research on Brazilians with BRONJ between 2010 and 2019, indexed in PubMed/MEDLINE, Scopus, Web of Science and LILACS were reviewed. Age, sex, type and time of bisphosphonate intake, administration route, related diseases, region of the BRONJ, diagnostic criteria, staging, triggering factor and type of treatment were analyzed.

RESULTS: Fifteen articles on 128 subjects were included. Most patients were women (82.03\%); the mean age was 63 years. Intravenous zoledronic acid was mostly used (62.50\%), for breast cancer treatment (46.87\%). The main localization of BRONJ was the mandible (54.68\%), associated mainly with tooth extractions (45.98\%). The diagnostic criteria were clinical (100\%) and radiographic (89.06\%), mostly in stage II (68.08\%). The surgical treatments were sequestrectomy (37.50\%) and platelet-rich plasma (PRP) (36.71\%). Microbial control was done using chlorhexidine (93.75\%) and infection control using clindamycin (53.90\%). CONCLUSIONS: BRONJ had higher prevalence in Brazilian women receiving treatment for breast cancer and osteoporosis. The mandible was the region most affected with a moderate stage of BRONJ, particularly when there were histories of tooth extraction and peri-implant surgery. Sequestrectomy with additional drugs and surgical therapy was the treatment most accomplished.
\end{abstract}

\section{INTRODUCTION}

Bisphosphonates (BPs) are drugs with oncological indication that have been used since $1960 .{ }^{1}$ They are currently indicated as therapy for multiple myeloma, malignant hypercalcemia, prevention of bone metastases and pathological fractures. ${ }^{2}$ BPs may also be prescribed for other diseases such as rheumatoid arthritis, osteoporosis and osteopenia. ${ }^{3-5}$

The mechanism of action of BPs consists of decreasing local vascular support and regulating bone metabolism, thereby reducing the action of osteoclasts and decreasing angiogenesis. Therefore, bone remodeling and deposition of physiological bone matrix are also affected. ${ }^{6,7}$ These effects on bone metabolism associated with local triggering factors, such as infection and tissue inflammation in the mouth, are named bisphosphonate-related osteonecrosis of the jaw (BRONJ). ${ }^{8}$ Among the main triggering factors of BRONJ are the following: exodontia, peri-implant surgery and traumas in the buccal mucosa. ${ }^{1,9-11}$ The clinical characteristics of BRONJ can include asymptomatic manifestations, severe pain, presence of infections and bone exposure. ${ }^{6}$

In 2014, there was a change in the nomenclature for this disease, to take into account its relationship with other drugs. The names currently used follow the pattern [medication]-related osteonecrosis of the jaw. This relates to the use of anti-resorptive and antiangiogenic medications, ${ }^{8,12-14}$ and more recent studies also mention the use of tyrosine kinase-inhibitor drugs and mammalian-target inhibitors of rapamycin. ${ }^{8-14}$

Although, as mentioned before, other medications relating to maxillary osteonecrosis do exist, BPs are still the most relevant drugs in relation to osteonecrosis of the jaws. ${ }^{8}$ The Brazilian 
population has high rates of breast and prostate cancer, ${ }^{15}$ and for this reason, BPs are highly indicated, which exposes these individuals to the risk of BRONJ.

The clinical profile of BRONJ and treatment protocols can vary according to specific demographic factors. Therefore, there is a need for population-specific studies. However, there are no studies in the literature reporting on the features of BRONJ and its treatment in Brazil.

\section{OBJECTIVE}

This integrative review aimed to determine the clinical profile of osteonecrosis of the jaw exclusively associated with bisphosphonate therapy in the Brazilian population.

\section{METHODS}

The guiding question of this review was: What are the clinical features of BRONJ in the Brazilian population that determine its clinical profile?

The inclusion criterion for this integrative review was that publications relating to Brazilian individuals would be included: these could include case reports, case series and clinical studies. Over the last decade, the nomenclature, staging and treatment method for BRONJ have undergone changes. This review considered articles either in English or in Portuguese that were published between January 2010 and April 2019. Articles that comprised review of the literature, laboratory analyses, letters to the editor, studies conducted on animal studies and research that did not involve Brazilians were excluded.

The variables selected were the following: age, sex, type of bisphosphonate used, duration of use of bisphosphonates until disease manifestation, route of administration, underlying disease that led to indication for drug use, oral region affected by BRONJ, clinical criteria for diagnosis of BRONJ, clinical staging according to the American Association of Oral and Maxillofacial Surgeons (AAOMS), ${ }^{8}$ local triggering factor and type of treatment. When data on any of these variables were absent, the study was not included in this review.

Four online databases were searched for articles: PubMed, Scopus, Web of Science and LILACS. PubMed, Scopus and Web of Science are international databases that have a search filter for the nationality of the articles, and this was used after the initial search. LILACS is a Latin American database with descriptors in the English and Portuguese language. We used the country identification tool for the Scopus, Web of Science and LILACS, and for PubMed we add the descriptor "Brazil". The descriptors entered in the databases are described below.

1. "osteonecrosis" and "bisphosphonate" and "Brazil" for PubMed.

2. "osteonecrosis" and "bisphosphonate" for Web of Science and Scopus
3. "osteonecrosis" and "bisphosphonate" and "bisphosphonate or "diphosphonate" and "osteonecrosis" for Lilacs.

The selection of the articles that were assessed in full for the analysis on each of the variables of this review is described in Figure 1.

\section{RESULTS}

Fifteen studies were included (Figure 1). ${ }^{4,5,9,16-26}$ A total of 141 different areas of BRONJ were reported in 128 individuals, of

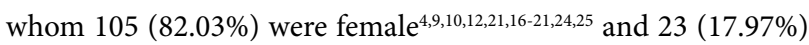
were male. ${ }^{5,9,10,16-19,25}$ The subjects' minimum age was 38 years and maximum age was 90 years, with a mean of $63.34 \pm 34.87$ years.

The commonest diagnoses relating to BRONJ were: breast cancer, in 60 individuals ${ }^{9,10,12,16,17,19,21}$ (46.87\%); osteoporosis, ${ }^{10,17-19,23-26}$ in 25 (19.53\%); multiple myeloma, ${ }^{5,9,16,17,20}$ in $16(12.50 \%)$; and prostate cancer, ${ }^{9,10,16,18,19}$ in 16 (12.50\%) (Table 1). ${ }^{4,5,9,10,12,16-21,23-26}$

The types of BPs most related to BRONJ were: zoledronic acid $^{9,10,12,16-21,26}$ (ZOE), in a dose of $4 \mathrm{mg}$ intravenously, in 80 cases (62.50\%); and alendronate $e^{4,10,17-19,23-26}$ (ALE) in a dose of between 70 and $90 \mathrm{mg}$ orally, in 26 cases (20.31\%). However, other BPs such as pamidronate ${ }^{5,9,10,16,19}$ (PAM) (10.93\%), an association of ZOE and $\mathrm{PAM}^{10,19,26}$ in six cases $(4.68 \%)$ and an association of $\mathrm{ZOE}$ and $\mathrm{ALE}^{10,26}$ in two cases (1.56\%) were also used. The duration of BP use until a manifestation of BRONJ ranged from 5 to 15 months, with a mean of $45.8 \pm 39.7$ months. ${ }^{4,5,9,16-26}$

Regarding the location of the BRONJ, the mandible was the most affected by BRONJ, ${ }^{4,9,10,16-20,25}$ in 70 cases (54.68\%), followed by the maxilla $a^{9,10,12,16-19,21,23-26}$ in 52 cases (40.62\%). Less frequent

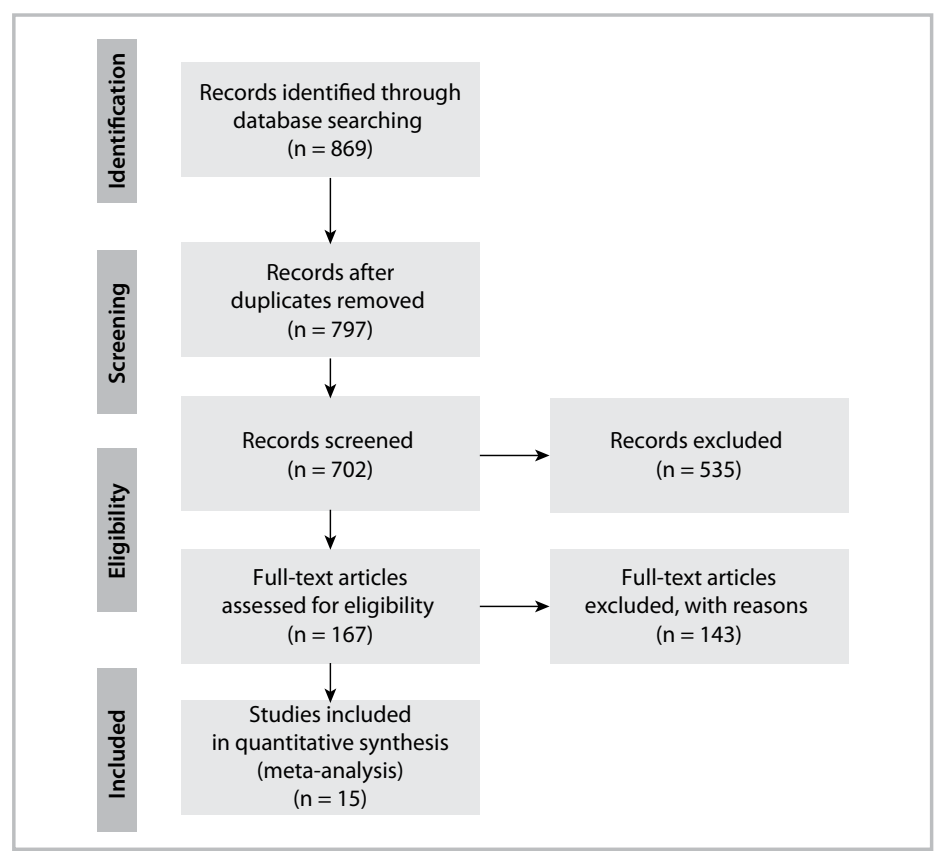

Figure 1. Flow diagram of the studies included in the integrative review 
Table 1. Reports on Brazilian individuals with bisphosphonate-related osteonecrosis of the jaw

\begin{tabular}{|c|c|c|c|c|c|c|c|}
\hline Authors & Title & $\begin{array}{c}\text { Type of } \\
\text { bisphosphonate }\end{array}$ & $\begin{array}{c}\text { No. of } \\
\text { individuals }\end{array}$ & Gender & Age & $\begin{array}{l}\text { Duration of } \\
\text { bisphosphonate } \\
\text { intake (months) }\end{array}$ & Related diseases \\
\hline $\begin{array}{l}\text { Antonini } \\
\text { et al. }{ }^{21}\end{array}$ & $\begin{array}{l}\text { Management of osteonecrosis of } \\
\text { the jaws in patients with history of } \\
\text { bisphosphonates therapy. }\end{array}$ & ZOE (1/1) & 1 & Female & 72 & 36 & BC \\
\hline $\begin{array}{l}\text { Conte-Neto } \\
\text { et al. }{ }^{4}\end{array}$ & $\begin{array}{l}\text { Oral bisphosphonate-related } \\
\text { osteonecrosis of the jaws in } \\
\text { rheumatoid arthritis patients: a } \\
\text { critical discussion and two case } \\
\text { reports. }\end{array}$ & $\operatorname{ALE}(2 / 2)$ & 2 & Female $(2 / 2)$ & $58-68$ & $84-96$ & $\mathrm{RA}(2 / 2)$ \\
\hline $\begin{array}{l}\text { Curi } \\
\text { et al. }{ }^{9}\end{array}$ & $\begin{array}{l}\text { Bisphosphonate-related } \\
\text { osteonecrosis of the jaws--an initial } \\
\text { case series report of treatment } \\
\text { combining partial bone resection } \\
\text { and autologous platelet-rich plasma. }\end{array}$ & $\begin{array}{l}\text { ZOE }(21 / 25) \\
\operatorname{PAM}(4 / 25)\end{array}$ & 25 & $\begin{array}{l}\text { Female }(20 / 25) \\
\text { Male }(5 / 25)\end{array}$ & $42-85$ & $12-84$ & $\begin{array}{l}\mathrm{BC}(14 / 25) \\
\mathrm{MM}(7 / 25) \\
\mathrm{PC}(4 / 25)\end{array}$ \\
\hline $\begin{array}{l}\text { Farias } \\
\text { et al. }{ }^{17}\end{array}$ & $\begin{array}{l}\text { Clinical and image findings } \\
\text { in bisphosphonate-related } \\
\text { osteonecrosis of the jaws. }\end{array}$ & $\begin{array}{l}\operatorname{ZOE}(5 / 7) \\
\operatorname{ALE}(2 / 7)\end{array}$ & 7 & $\begin{array}{c}\text { Female }(6 / 7) \\
\text { Male }(1 / 7)\end{array}$ & $42-76$ & $36-156$ & $\begin{array}{l}\mathrm{BC}(2 / 7) \\
\mathrm{OP}(2 / 7) \\
\mathrm{MM}(1 / 7) \\
\operatorname{LVC}(1 / 7) \\
\mathrm{GCC}(1 / 7)\end{array}$ \\
\hline $\begin{array}{l}\text { Rabelo } \\
\text { et al. }{ }^{18}\end{array}$ & $\begin{array}{l}\text { Bisphosphonate-Related } \\
\text { Osteonecrosis of the Jaws and Its } \\
\text { Array of Manifestations. }\end{array}$ & $\begin{array}{l}\operatorname{ZOE}(4 / 8) \\
\operatorname{ALE}(4 / 8)\end{array}$ & 8 & $\begin{array}{c}\text { Female }(6 / 8) \\
\text { Male }(2 / 8)\end{array}$ & $53-76$ & $5-72$ & $\begin{array}{l}\mathrm{OP}(4 / 8) \\
\mathrm{BC}(2 / 8) \\
\mathrm{PC}(1 / 8) \\
\mathrm{PD}(1 / 8)\end{array}$ \\
\hline Lopes et al. ${ }^{10}$ & $\begin{array}{l}\text { Surgical Therapy for } \\
\text { Bisphosphonate-Related } \\
\text { Osteonecrosis of the Jaw: Six-Year } \\
\text { Experience of a Single Institution. }\end{array}$ & $\begin{array}{c}\text { ZOE }(22 / 33) \\
\text { ZOE + PAM }(5 / 33) \\
\operatorname{PAM}(3 / 33) \\
\operatorname{ALE}(2 / 33) \\
\text { ZOE }+ \text { ALE }(1 / 33)\end{array}$ & 33 & $\begin{array}{c}\text { Female }(25 / 33) \\
\text { Male }(8 / 33)\end{array}$ & $39-83$ & $26-120$ & $\begin{array}{l}\text { BC (18/33) } \\
\text { MM (4/33) } \\
\text { PC (4/33) } \\
\text { LC (4/33) } \\
\text { OP }(2 / 33) \\
\text { KC ( } 1 / 33)\end{array}$ \\
\hline $\begin{array}{l}\text { Heggendorn } \\
\text { et al. }{ }^{20}\end{array}$ & $\begin{array}{l}\text { Bisphosphonate-related } \\
\text { osteonecrosis of the jaws: Report of } \\
\text { a case using conservative protocol. }\end{array}$ & ZOE (1/1) & 1 & Female & 52 & 22 & MM \\
\hline Maluf et al. ${ }^{12}$ & $\begin{array}{l}\text { Surgery Combined with LPRF in } \\
\text { Denosumab Osteonecrosis of the } \\
\text { Jaw: Case Report. }\end{array}$ & ZOE (1/1) & 1 & Female & 79 & 36 & $B C$ \\
\hline $\begin{array}{l}\text { Momesso } \\
\text { et al. }{ }^{23}\end{array}$ & $\begin{array}{c}\text { Successful use of lower-level } \\
\text { laser therapy in the treatment of } \\
\text { medication-related osteonecrosis of } \\
\text { the jaw. }\end{array}$ & $\operatorname{ALE}(1 / 1)$ & 1 & Female & 65 & 60 & OP \\
\hline $\begin{array}{l}\text { de Oliveira } \\
\text { Ruellas } \\
\text { et al. }{ }^{24}\end{array}$ & $\begin{array}{l}\text { Managing bisphosphonate-related } \\
\text { osteonecrosis of the jaws with } \\
\text { xenografts: a case report. }\end{array}$ & $\operatorname{ALE}(1 / 1)$ & 1 & Female & 69 & 120 & OP \\
\hline
\end{tabular}


Table 1. Continuation

\begin{tabular}{|c|c|c|c|c|c|c|c|}
\hline Authors & Title & $\begin{array}{c}\text { Type of } \\
\text { bisphosphonate }\end{array}$ & $\begin{array}{c}\text { No. of } \\
\text { individuals }\end{array}$ & Gender & Age & $\begin{array}{l}\text { Duration of } \\
\text { bisphosphonate } \\
\text { intake (months) }\end{array}$ & Related diseases \\
\hline $\begin{array}{l}\text { Fernando } \\
\text { de Almeida } \\
\text { Mourão } \\
\text { et al. } .^{25}\end{array}$ & $\begin{array}{l}\text { The use of Platelet-rich Fibrin in the } \\
\text { management of medication-related } \\
\text { osteonecrosis of the jaw: A case } \\
\text { series. }\end{array}$ & $\operatorname{ALE}(11 / 11)$ & 11 & $\begin{array}{c}\text { Female }(9 / 11) \\
\text { Male }(2 / 11)\end{array}$ & $38-84$ & $36-84$ & OP \\
\hline $\begin{array}{l}\text { Santos } \\
\text { et al. } .^{26}\end{array}$ & $\begin{array}{l}\text { Extensive osteonecrosis of the } \\
\text { maxilla caused by bisphosphonates: } \\
\text { Report of a rare case. }\end{array}$ & $\mathrm{ZOE}+\mathrm{ALE}(1 / 1)$ & 1 & Female & 52 & 144 & OP \\
\hline Total & & $\begin{array}{c}\text { ZOE } 80(62.50 \%) \\
\text { ALE } 26(20.31 \%) \\
\text { PAM } 14(10.93 \%) \\
\text { ZOE + PAM } 6 \\
(4.68 \%) \\
\text { ZOE + ALE } 2 \\
(1.56 \%)\end{array}$ & $\begin{array}{c}128 \\
(100 \%)\end{array}$ & $\begin{array}{l}\text { Female } 105 \\
\text { (82.03\%) } \\
\text { Male 23 } \\
(17.97 \%)\end{array}$ & $\begin{array}{c}63.34 \\
\text { (mean) }\end{array}$ & $\begin{array}{l}45 \text { months } \\
\text { (mean) }\end{array}$ & $\begin{array}{c}\text { BC } 60 \text { (46.87\%) } \\
\text { OP } 25(19.53 \%) \\
\text { MM } 16 \\
(12.50 \%) \\
\text { PC } 16(12.50 \%) \\
\text { LC } 5(3.90 \%) \\
\text { RA } 2(1.56 \%) \\
\text { KC } 1(0.78 \%) \\
\text { PD } 1(0.78 \%) \\
\text { GCC } 1(0.78 \%) \\
\text { LVC } 1(0.78 \%)\end{array}$ \\
\hline
\end{tabular}

$\mathrm{ZOE}=$ zoledronate; $\mathrm{PAM}=$ pamidronate; $\mathrm{ALE}=$ alendronate; $\mathrm{BC}=$ breast cancer; $\mathrm{MM}=$ multiple myeloma; $\mathrm{PC}=$ prostate cancer; $\mathrm{OP}=$ osteoporosis, $\mathrm{LC}=$ lung cancer; $\mathrm{RA}=$ rheumatoid arthritis; $\mathrm{KC}=$ kidney cancer; $\mathrm{PD}=$ Paget's disease; GCCA = giant-cell cancer; $\mathrm{LVC}=$ liver cancer.

manifestations were in both jaws, ${ }^{16,17,19}$ the palatine torus ${ }^{10}$ and the mylohyoid region ${ }^{17}$ (Table 2). ${ }^{4,5,9,10,12,16-21,23-26}$

The main diagnostic criterion was clinical evaluation of bone exposure, ${ }^{4,5,9,16-26}$ which was found to be present in $100 \%$ of the cases. The most common complementary examinations were panoramic radiographs, ${ }^{4,5,9,10,16-20,23,25}$ in 114 cases (89.06\%); and conebean computed tomography, ${ }^{4,5,12,16,19,21,24-26}$ in 52 cases (40.62\%). In contrast, the histopathological evaluation ${ }^{4,21}$ was present only in three cases (2.34\%) (Table 2)., ${ }^{4,59,10,12,16-21,23-26}$

The local trigger factors for BRONJ were the following: tooth extraction, ${ }^{5,9,10,12,16-19,25,26}$ in 63 individuals (45.98\%); implant placement, ${ }^{9,17,19,23,25}$ in 19 (13.86\%); prosthetic trauma, ${ }^{9,10,16,20}$ in 19 (13.86\%); and spontaneous manifestation, ${ }^{9,10,16-19,21}$ in 19 (13.86\%). Other local factors are considered in Table 2 . $^{4,5,9,10,12,16-21,23-26}$

According to the AAOMS classification, three individuals $(2.12 \%)$ presented stage $0^{17,18}$ of BRONJ; 16 cases (11.34\%) had stage $\mathrm{I} ;{ }^{9,16-18} 96$ cases $(68.08 \%$, i.e. the majority) presented stage II; $;^{4,5,9,10,16-20,23,25}$ and 26 cases (18.43\%) had stage III ${ }^{9,10,12,16,18,21,24,26}$ (Table 2). ${ }^{4,5,9,10,12,16-21,23-26}$

There was a lack of detailed information about the types of treatment and management used in these cases reported from Brazilian populations. The treatment most reported was sequestrectomy, ${ }^{4,5,10}$ in 48 cases (37.50\%); while platelet-rich plasma ${ }^{9,16,19,21,25}$ was used to complement surgery, in 47 cases (36.71\%). Other treatments, such as bone resection with or without curettage, ${ }^{9,16,19,21,24-26}$ tooth extractions with debridement of the necrotic bone, ${ }^{4,9}$ debridement alone $e^{12,17,18}$ or even low power laser therapy, are described in Table 2. ${ }^{4,5,9,10,12,16-21,23-26}$
The topical medication most used for treatment of BRONJ was chlorhexidine solution, ${ }^{4,5,9,16,17,19-26}$ in 120 individuals (93.75\%). The systemic medication used consisted of antibiotic therapy with clindamycin in 69 cases (53.90\%) and amoxicillin in four cases $(3.12 \%) .{ }^{12,17,26}$ Other antibiotics were also reported to have been used as part of BRONJ treatments but without any detailed description. Thus, for this reason, they were not included in this review.

\section{DISCUSSION}

The cases of BRONJ in Brazil showed that 103 individuals (80.47\%) received BPs intravenously as part of their cancer treatment. The other 25 (19.53\%) received BPs orally as osteoporosis treatment. In North American populations, similar results were observed, i.e. BRONJ developed mainly in individuals who were undergoing oncological treatment intravenously. ${ }^{27,28}$ Use of this administration route increases the risk of BRONJ one hundredfold, compared with the oral route. ${ }^{8}$

The oral route for BPs is more used for controlling osteoporosis: not only in Brazil ${ }^{4,5,9,16-26}$ but also in the United States. ${ }^{27,28}$ Among BPs, ALE is the BP that is most associated with BRONJ. ${ }^{4,5,9,16-2}$ In Europe, individuals with rheumatoid arthritis have higher incidence of BRONJ than individuals with osteoporosis, since ALE is the treatment of choice and its use is prolonged. ${ }^{29-31}$

In addition to the type of diagnosis of the disease and type of BP, surgical manipulation of the jaws ${ }^{19,32}$ accounts for $60 \%$ of the local trigger factors for developing BRONJ. ${ }^{32-34}$ Thus, in these Brazilian cases, the triggering factor most reported was tooth dental extraction (45.98\%), 
Table 2. Clinical features of bisphosphonate-related osteonecrosis of the jaw (BRONJ)

\begin{tabular}{|c|c|c|c|c|c|c|c|}
\hline Authors & Title & Region of BRONJ & $\begin{array}{l}\text { No. of } \\
\text { cases of } \\
\text { BRONJ }\end{array}$ & Triggering factor & $\begin{array}{l}\text { Diagnostic } \\
\text { criteria }\end{array}$ & $\begin{array}{l}\text { AAOMS } \\
\text { classification } \\
\text { (stage) }\end{array}$ & Type of treatment \\
\hline $\begin{array}{l}\text { Antonini } \\
\text { et al. }{ }^{21}\end{array}$ & $\begin{array}{l}\text { Management of osteonecrosis of } \\
\text { the jaws in patients with history } \\
\text { of bisphosphonates therapy. }\end{array}$ & $\begin{array}{l}\text { Post. right reg. } \\
\text { maxilla }\end{array}$ & 1 & Spontaneous & $\begin{array}{l}\text { CLN, TOMO } \\
\text { and HPT }\end{array}$ & III (1/1) & $\begin{array}{l}\text { HBO therapy, RES, } \\
\text { PRP, CFLX, CHX, DH }\end{array}$ \\
\hline $\begin{array}{l}\text { Conte-Neto } \\
\text { et al. }{ }^{4}\end{array}$ & $\begin{array}{l}\text { Oral bisphosphonate-related } \\
\text { osteonecrosis of the jaws in } \\
\text { rheumatoid arthritis patients: a } \\
\text { critical discussion and two case } \\
\text { reports. }\end{array}$ & $\begin{array}{l}\text { Right reg. } \\
\text { mandible; left reg. } \\
\text { mandible }\end{array}$ & 2 & $\begin{array}{c}\text { Trauma; } \\
\text { periodontitis }\end{array}$ & $\begin{array}{l}\text { CLN, TOMO, } \\
\text { and HPT; } \\
\text { CLN, RAD } \\
\text { and TOMO }\end{array}$ & II $(2 / 2)$ & $\begin{array}{c}\text { AT Clin. CHX, } \\
\text { IR, SEQ, AdDEB; } \\
\text { CHX, AT Clav. EXT, } \\
\text { AdDEB. }\end{array}$ \\
\hline $\begin{array}{l}\text { Curi } \\
\text { et al. }{ }^{9}\end{array}$ & $\begin{array}{l}\text { Bisphosphonate-related } \\
\text { osteonecrosis of the jaws--an } \\
\text { initial case series report of } \\
\text { treatment combining partial } \\
\text { bone resection and autologous } \\
\text { platelet-rich plasma. }\end{array}$ & $\begin{array}{c}\text { Mandible }(8 / 25) ; \\
\text { maxilla }(7 / 25)\end{array}$ & 25 & $\begin{array}{c}\text { Extraction } \\
(14 / 25) ; \text { prosthetic } \\
\text { trauma }(7 / 25) ; \\
\text { implant }(2 / 25) ; \\
\text { spontaneous } \\
(2 / 25)\end{array}$ & $\begin{array}{l}\text { CLN and } \\
\text { RAD }\end{array}$ & $\begin{array}{l}\text { I }(3 / 25) \\
\text { II }(15 / 25) \\
\text { III }(7 / 25)\end{array}$ & $\begin{array}{l}\text { CHX, AT; AT Clin, } \\
\text { RES, AdDEB, PRP; } \\
\text { EXT AdDEB, PRP }\end{array}$ \\
\hline $\begin{array}{l}\text { Farias } \\
\text { et al. }{ }^{17}\end{array}$ & $\begin{array}{l}\text { Clinical and image findings } \\
\text { in bisphosphonate-related } \\
\text { osteonecrosis of the jaws. }\end{array}$ & $\begin{array}{l}\text { Post. bilateral reg. } \\
\text { mandible }(2 / 7) ; \\
\text { right myeloid reg. } \\
(1 / 7) ; \text { both post. } \\
\text { reg. jaws ( } 1 / 7) ; \\
\text { both post. left reg. } \\
\text { jaws }(1 / 7) ; \text { ant. reg. } \\
\text { maxilla ( } 2 / 7)\end{array}$ & 7 & $\begin{array}{c}\text { Extraction }(4 / 7) ; \\
\text { Extraction }+ \\
\text { Implant. (1/7); } \\
\text { spontaneous (2/7) }\end{array}$ & $\begin{array}{l}\text { CLN and } \\
\text { RAD }\end{array}$ & $\begin{array}{l}0(2 / 7) \\
\text { I (1/7) } \\
\text { II (4/7) }\end{array}$ & $\begin{array}{c}\mathrm{DEBL}+\mathrm{AT} \text { Amox } \\
+\mathrm{CHX}\end{array}$ \\
\hline $\begin{array}{l}\text { Mathias } \\
\text { Duarte } \\
\text { et al. }{ }^{19}\end{array}$ & $\begin{array}{c}\text { Bisphosphonate-related } \\
\text { osteonecrosis of the jaws: } \\
\text { analysis of a case series at a } \\
\text { dental school. }\end{array}$ & $\begin{array}{l}\text { Mandible }(8 / 13) ; \\
\text { maxilla }(4 / 13) ; \\
\text { both jaws }(1 / 13)\end{array}$ & 13 & $\begin{array}{l}\text { Extraction }(7 / 13) ; \\
\text { implant }(2 / 13) ; \\
\text { periodontitis } \\
(2 / 13) ; \\
\text { spontaneous } \\
(2 / 13)\end{array}$ & $\begin{array}{l}\text { CLN, RAD } \\
\text { and TOMO }\end{array}$ & II (13) & $\begin{array}{l}\text { CHX, AT Clin. } \\
(3 / 13) ; \operatorname{RES}(4 / 13) ; \\
\text { PRP }(6 / 13)\end{array}$ \\
\hline $\begin{array}{l}\text { Zanata } \\
\text { et al. } .^{5}\end{array}$ & $\begin{array}{l}\text { Bisphosphonate-related } \\
\text { osteonecrosis of the jaw in } \\
\text { patient affected by multiple } \\
\text { myeloma: A case report. }\end{array}$ & Post. reg. mandible & 1 & Extraction & $\begin{array}{l}\text { CLN, RAD } \\
\text { and TOMO }\end{array}$ & II (1/1) & 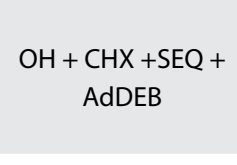 \\
\hline Lopes et al. ${ }^{10}$ & $\begin{array}{c}\text { Surgical Therapy for } \\
\text { Bisphosphonate-Related } \\
\text { Osteonecrosis of the Jaw: } \\
\text { Six-Year Experience of a Single } \\
\text { Institution. }\end{array}$ & $\begin{array}{l}\text { Post. reg. } \\
\text { mandible. (19/46); } \\
\text { post. reg. maxilla } \\
\text { (16/46); Ant. reg. } \\
\text { mandible (5/46); } \\
\text { Ant. reg. maxilla. } \\
\text { (3/46); mandibular } \\
\text { torus (2/46); both } \\
\text { jaws }(1 / 46)\end{array}$ & 46 & $\begin{array}{c}\text { Extraction }(16 / 46) ; \\
\text { periodontitis }+ \\
\text { periimplantitis } \\
\text { (9/46); prosthetic } \\
\text { trauma }(8 / 46) ; \\
\text { spontaneous } \\
(8 / 46) ; \text { implant } \\
\text { (3/46); palatine } \\
\text { torus trauma }(2 / 46)\end{array}$ & $\begin{array}{l}\text { CLN and } \\
\text { RAD }\end{array}$ & $\begin{array}{l}\text { II (37/46) } \\
\text { III }(9 / 46)\end{array}$ & $\begin{array}{l}\text { Ad DEB; SEQ; AT } \\
\text { Clin + AT + CHX }\end{array}$ \\
\hline
\end{tabular}


Table 2. Continuation

\begin{tabular}{|c|c|c|c|c|c|c|c|}
\hline Authors & Title & Region of BRONJ & $\begin{array}{l}\text { No. of } \\
\text { cases of } \\
\text { BRONJ }\end{array}$ & Triggering factor & $\begin{array}{l}\text { Diagnostic } \\
\text { criteria }\end{array}$ & $\begin{array}{l}\text { AAOMS } \\
\text { classification } \\
\text { (stage) }\end{array}$ & Type of treatment \\
\hline $\begin{array}{l}\text { Heggendorn } \\
\text { et al. }{ }^{20}\end{array}$ & $\begin{array}{l}\text { Bisphosphonate-related } \\
\text { osteonecrosis of the jaws: } \\
\text { Report of a case using } \\
\text { conservative protocol. }\end{array}$ & $\begin{array}{l}\text { Post. left. reg. } \\
\text { mandible }\end{array}$ & 1 & Prosthetic trauma & $\begin{array}{l}\text { CLN and } \\
\text { RAD }\end{array}$ & II (1/1) & CHX + LLLT \\
\hline $\begin{array}{l}\text { Maluf } \\
\text { et al. }{ }^{12}\end{array}$ & $\begin{array}{l}\text { Surgery Combined with LPRF in } \\
\text { Denosumab Osteonecrosis of } \\
\text { the Jaw: Case Report. }\end{array}$ & Ant. reg. maxilla & 1 & Extraction & $\begin{array}{l}\text { CLN and } \\
\text { TOMO }\end{array}$ & III $(1 / 1)$ & $\begin{array}{c}\text { DEB, LPRF, CHX, } \\
\text { Amox }+ \text { CLV, MTZ, } \\
\text { DH }\end{array}$ \\
\hline $\begin{array}{l}\text { Momesso } \\
\text { et al. } .^{23}\end{array}$ & $\begin{array}{l}\text { Successful use of lower-level } \\
\text { laser therapy in the treatment } \\
\text { of medication-related } \\
\text { osteonecrosis of the jaw. }\end{array}$ & $\begin{array}{l}\text { Post. right reg. } \\
\text { maxilla. }\end{array}$ & 1 & Implant & $\begin{array}{l}\text { CLN and } \\
\text { RAD }\end{array}$ & II (1/1) & $\mathrm{LLLT}+\mathrm{CHX}+\mathrm{Clin}$ \\
\hline $\begin{array}{l}\text { de Oliveira } \\
\text { Ruellas } \\
\text { et al. }{ }^{24}\end{array}$ & $\begin{array}{l}\text { Managing bisphosphonate- } \\
\text { related osteonecrosis of the jaws } \\
\text { with xenografts: a case report. }\end{array}$ & $\begin{array}{l}\text { Post. right reg. } \\
\text { maxilla. }\end{array}$ & 1 & $\begin{array}{l}\text { Odontogenic } \\
\text { infection }\end{array}$ & $\begin{array}{l}\text { CLN and } \\
\text { TOMO }\end{array}$ & III $(1 / 1)$ & $\begin{array}{c}\text { Ress, EXT, graft, } \\
\text { AdDEB, CFLX, MTZ, } \\
\text { CHX }\end{array}$ \\
\hline $\begin{array}{l}\text { Santos } \\
\text { et al. }{ }^{26} 2019\end{array}$ & $\begin{array}{c}\text { Extensive osteonecrosis } \\
\text { of the maxilla caused by } \\
\text { bisphosphonates: Report of a } \\
\text { rare case. }\end{array}$ & $\begin{array}{l}\text { Ant. and post. } \\
\text { bilateral reg. } \\
\text { maxilla }(1 / 1)\end{array}$ & 1 & Extraction & $\begin{array}{l}\text { CLN and } \\
\text { TOMO }\end{array}$ & III $(1 / 1)$ & $\begin{array}{c}\text { CHX, Amox + CLV, } \\
\text { RES, AdDEB }\end{array}$ \\
\hline Total & & $\begin{array}{c}\text { Post. reg. } \\
\text { mandible } 33 \\
(25.78 \%) ; \text { post. } \\
\text { reg. maxilla } 24 \\
\text { (18.75\%); ant. reg. } \\
\text { maxilla } 7 \text { (5.46\%); } \\
\text { ant. reg. mandible } \\
6 \text { (4.68\%); post. } \\
\text { reg. both jaws } 2 \\
\text { (1.56\%); palatine } \\
\text { torus } 2(1.56 \%) ; \\
\text { myeloid reg. } 1 \\
\text { (0.78\%); post. and } \\
\text { ant. reg. both jaws } \\
1 \text { (0.78\%) }\end{array}$ & $\begin{array}{c}141 \\
(100 \%)\end{array}$ & $\begin{array}{c}\text { Extraction } 63 \\
(45.98 \%) ; \text { implant } \\
19(13.86 \%) ; \\
\text { prosthetic } \\
\text { trauma } 19 \\
(13.86 \%) ; \\
\text { spontaneous } \\
19(13.86 \%) ; \\
\text { periodontitis } \\
13(10.65 \%) ; \\
\text { palatine torus } \\
\text { trauma } 2(1.45 \%) ; \\
\text { trauma } 1(0.73 \%) ; \\
\text { odontogenic } \\
\text { infection } 1 \\
(0.72 \%) \\
137(100 \%)\end{array}$ & $\begin{array}{c}\text { CLN } \\
128(100 \%) ; \\
\text { RAD } 114 \\
(89.06 \%) ; \\
\text { TOMO } 52 \\
(40.62 \%) ; \\
\text { HPT } 3 \\
(2.34 \%) \\
128(100 \%)\end{array}$ & $\begin{array}{c}\text { Stage 0: } 3 \\
(2.12 \%) ; \\
\text { stage I: } 16 \\
(11.34 \%) ; \\
\text { stage II: } 96 \\
\text { (68.08\%); } \\
\text { Stage III: } 26 \\
\text { (18.43\%) } \\
141(100 \%)\end{array}$ & $\begin{array}{c}\text { CHX } 120(93.75 \%) ; \\
\text { Clin } 69(53.90 \%) ; \\
\text { AT } 54(42.18 \%) ; \\
\text { SEQ + adDEB } 48 \\
(37.50 \%) ; \text { PRP } \\
47(36.71 \%) ; \\
\text { EXT + adDEB } 26 \\
(20.31 \%) ; \text { LLLT } \\
16(12.50 \%) ; \\
\text { RES + AdDEB } 13 \\
(10.15 \%) ; \text { Amox } \\
4(3.12 \%) ; \text { AD } \\
3(2.34 \%) ; \text { DH } \\
2(1.56 \%) ; \text { IR } 1 \\
(0.78 \%) ; \text { LPRF } 1 \\
(0.78 \%) ; \text { HBO } 1 \\
(0.78 \%) \\
128(100 \%)\end{array}$ \\
\hline
\end{tabular}

AT = other antibiotics; $\mathrm{CHX}=$ chlorhexidine; Ext = extraction; $\mathrm{AdDEB}=$ additional debridement; $\mathrm{SEQ}=$ sequestrectomy; $\mathrm{RES}=$ resection; IR = implant removal; $\mathrm{CLN}=$ clinical; $\mathrm{RAD}=$ radiographic; $\mathrm{TOMO}=$ tomographic; $\mathrm{HPT}=$ histopathologically; $\mathrm{DEBL}=$ local debridement; Reg. = region; $\mathrm{PRP}=$ platelet-rich plasma; $\mathrm{LLLT}=$ low-level laser therapy; Post. = posterior; $\mathrm{Ant}=$ anterior; $\mathrm{OH}=$ oral hygiene guidance; $\mathrm{Clin}=$ clindamycin; $\mathrm{AD}=$ abscess drainage; $\mathrm{Amox}=\mathrm{amoxicillin}$; $M T Z=$ metronidazole; $C F L X=$ cefalexin; $D H=$ drug holiday; $\mathrm{HBO}=$ hyperbaric oxygen therapy; $\mathrm{LPRF}=$ leukocyte-platelet-rich fibrin.

especially in individuals using ZOE (62.50\%). Tooth extraction was also reported to be the main local factor that triggered BRONJ in North American, ${ }^{27}$ European $^{31,34-39}$ and Asian ${ }^{40-43}$ populations.

Among the triggering factors for BRONJ, implant surgery still remains a matter of controversy in the literature. ${ }^{8} \mathrm{~A}$ systematic review found that there was no evidence to demonstrate safety in performing dentoalveolar surgical procedures such as placement of dental implants in individuals exposed to BPs. Therefore, such procedures should be considered to be local risk factors. ${ }^{44}$ In one Brazilian population, implant placement was considered to be the 
second most prevalent trigger factor (13.86\%), with epidemiological values similar to those of a European population (13.50\%). ${ }^{35}$ Placement of dental implants in individuals who are using BPs presents local and systemic risk factors for development of BRONJ, regardless of the route of $\mathrm{BP}$ administration. Hence, these patients should not be treated in a conventional manner. The imminent risk of BRONJ and the risk of failure of peri-implant treatment should be always considered in drawing up the treatment plan. Patients should always be made aware of their systemic and dental condition.

Among the jaw bones, the chance of developing BRONJ is twice as high in the mandible as in the maxilla. ${ }^{8,32,42,45}$ In the present review, we observed greater involvement of the mandible, i.e. similar to the findings in North American, ${ }^{27,30,46}$ European ${ }^{31,34,35,37-39,47,48}$ and Asian ${ }^{40}$ ${ }^{43}$ populations. Despite the lack of detailed information regarding the location of BRONJ that was seen in the present review, we found that the posterior region of the mandible was the most involved, which coincided with findings from the rest of the world's population. $^{31,34,35,37-43,47,48}$ Therefore, in all cases, bone manipulation must be done in a precise, fast and atraumatic manner.

The fact that women have been found to be more affected by BRONJ, both in Brazil and in the rest of the world, ${ }^{27-31,34-43,47-50}$ may be related to administration of BPs after the menopause and to high incidence of breast cancer and osteoporosis. In Brazil, under these two conditions, there is an indication for using BPs. In the present study, breast cancer and osteoporosis were the underlying diseases that led to the highest prevalence of BRONJ, and similar results were found in European populations. ${ }^{36,38}$ Although the prevalence of BRONJ has mainly been associated with occurrences of breast cancer in the United States, multiple myeloma is the second most prevalent disease related to this oral complication..$^{27,34,38,46}$ The South Korean population is the only population in which BRONJ is more related to osteoporosis than to other underlying diseases. ${ }^{42,43}$

Therefore, it is important for medical specialists such as mastologists and/or gynecologists to be aware that patients who use $\mathrm{BPs}$ are at greater risk of developing BRONJ. One preventive measure could be to refer patients for dental assessment, before or during the first months of prescription of BPs, in order to eliminate some foci of infection that can expose these patients to the risk of developing BRONJ.

This integrative review identified that the majority of the Brazilian cases were diagnosed during stage 2 of BRONJ, and this is similar to findings from other countries. ${ }^{30,31,36-38,41-43,46,48}$ High prevalences of other stages are unusual, but when this occurs, it is usually stage 3 , in which there is involvement of adjacent structures such as the maxillary sinus or occurrence of pathological fracture of the mandible..$^{35,47-49}$ In the present review, stage 3 had the second highest prevalence, affecting $18.43 \%$ of this Brazilian population.

The diagnosis of BRONJ in these Brazilian cases was clinical in all of them. Since 2014, AAOMS has recommended the use of complementary imaging tests to finalize the staging and evaluate possible bone alterations that can precede BRONJ. Despite this recommendation for concomitant use of computed tomography (CT) as the most appropriate examination, the present review identified that only $40.62 \%$ of the cases were diagnosed by means of cone beam computed tomography (CBCT). This suggests not only that there is probably a lack of knowledge of indication of $3 \mathrm{D}$ imaging such as CBCT to perform better examinations, but also that there is a lack of local resources or else that these examinations have a high cost. The radiographic evaluation criterion was not included in this integrative review because of the lack of detailed information in the studies selected.

In addition, it is important to mention that, although AAOMS recommends that BRONJ should be diagnosed using clinical and imaging methods, we would emphasize that there is a need to make differential diagnoses in relation to other lesions with clinical signs of bone exposure, such as bone metastases and clinical manifestations of multiple myeloma in the jaw through histopathological analysis. ${ }^{50-52}$ Nonetheless, such lesions have been found to be very scarce, accounting for only $2.34 \%$.

The etiology and progression of BRONJ are related to infection and inflammation. ${ }^{8}$ In these Brazilian cases, sequestrectomy, resection and curettage were used, almost always in association with chlorhexidine mouthwashes and antibiotic therapy when necessary. In this last case, clindamycin was the main antibiotic selected, while other antibiotics like amoxicillin, tetracycline and metronidazole were also used but less frequently. Some studies have reported that the penicillin group was the first choice among antibiotics in Europe, ${ }^{34,35,47}$ and clarithromycin in Asia. ${ }^{40}$

Among the types of treatment mentioned earlier, surgical treatment is widely used in different populations around the world. ${ }^{29,34,41,46,47,53}$ Regardless of the type of surgical approach used, debridement or sequestrectomy until accessing the bleeding bone is recommended for improving the chances of success in the treatment. ${ }^{38}$

The limitation of the present study was its inability to provide detailed information about the location of BRONJ, type and dose of medications, radiographic features, biopsy and follow-up because of the lack of detailed information in the studies selected. In addition, there were no randomized studies or investigations on BRONJ in Brazilian populations. For this reason, we suggest that such studies need to be conducted and need to provide detailed information, as mentioned earlier.

\section{CONCLUSION}

The manifestation of BRONJ in this Brazilian population was greatest in the mandibles of younger females, with greater associations with breast cancer and osteoporosis. The major risk factor was previous exodontia, and BRONJ was diagnosed mainly 
in the intermediate staging (II). Surgical intervention was the treatment most commonly used among these Brazilian patients. This review identified greater use of chlorhexidine solution and prescription of clindamycin as the first-choice antibiotic therapy. PRP was the complementary therapy most used.

\section{REFERENCES}

1. Drake MT, Clarke BL, Khosla SM. Bisphosphonates: mechanism of action and role in clinical practice. Mayo Clin Proc. 2008;83(9):1032-45. PMID: 18775204; doi: 10.4065/83.9.1032.

2. Duarte LF, Alonso K, Basso EC, Dib LL. Surgical treatment of bisphosphonate-related osteonecrosis of the jaws with the use of buccal fat pad: case report. Braz Dent J. 2015;26(3):317-20. PMID: 26200161; doi: 10.1590/0103-6440201301918.

3. Miniello TG, Araújo JP, Lopes RN, Alves FA. Osteonecrosis related to once-yearly zoledronic acid treatment in an osteoporotic patient after dental implant. Braz Dent J. 2015;26(1):86-8. PMID: 25672391; doi: 10.1590/0103-6440201300255.

4. Conte-Neto N, Bastos AS, Spolidorio LC, Marcantonio RA, Marcantonio E Jr. Oral bisphosphonate-related osteonecrosis of the jaws in rheumatoid arthritis patients: a critical discussion and two case reports. Head Face Med. 2011;7:7. PMID: 21524309; doi: 10.1186/1746160X-7-7.

5. Zanata A, Felin GC, De Bona MC, Sawazaki R, De Conto F. Osteonecrose mandibular associada ao uso de bisfosfonato de sódio em paciente com mieloma múltiplo [Bisphosphonate-related osteonecrosis of the jaw in patient affected by multiple myeloma: A case report]. Revista Portuguesa de Estomatologia, Medicina Dentária e Cirurgia Maxilofacial. 2014;55(2):115-20. doi: 10.1016/j.rpemd.2014.04.002.

6. Marx RE. Pamidronate (Aredia) and zoledronate (Zometa) induced avascular necrosis of the jaws: a growing epidemic. J Oral Maxillofac Surg. 2003;61(9):1115-7. PMID: 12966493; doi: 10.1016/S02782391(03)00720-1.

7. Ruggiero SL, Mehrotra B, Rosenberg TJ, Engroff SL. Osteonecrosis of the jaws associated with the use of bisphosphonates: a review of 63 cases. J Oral Maxillofac Surg. 2004;62(5):527-34. PMID: 15122554; doi: 10.1016/j.joms.2004.02.004.

8. Ruggiero SL, Dodson TB, Fantasia J, et al. American Association of Oral and Maxillofacial Surgeons position paper on medicationrelated osteonecrosis of the jaw--2014 update. J Oral Maxillofac Surg. 2014;72(10):1938-56. PMID: 25234529; doi: 10.1016/j.joms.2014.04.031.

9. Curi MM, Cossolin GS, Koga DH, et al. Bisphosphonate-related osteonecrosis of the jaws--an initial case series report of treatment combining partial bone resection and autologous platelet-rich plasma. J Oral Maxillofac Surg. 2011;69(9):2465-72. PMID: 21763050; doi: 10.1016/j.joms.2011.02.078.

10. Lopes RN, Rabelo GD, Rocha AC, Carvalho PA, Alves FA. Surgical Therapy for Bisphosphonate-Related Osteonecrosis of the Jaw: Six-Year Experience of a Single Institution. J Oral Maxillofac Surg. 2015;73(7):128895. PMID: 25871903; doi: 10.1016/j.joms.2015.01.008.
11. Sverzurt CE, Sverzurt AT, de Matos FP, et al. Mandibular bisphosphonaterelated osteonecrosis after dental implant rehabilitation: a case report. Implant Dent. 2012;21(6):449-53. PMID: 23075811; doi: 10.1097/ ID.0b013e3182703c4f

12. Maluf G, Pinho MC, Cunha SR, Santos PS, Fregnani ER. Surgery Combined with LPRF in Denosumab Osteonecrosis of the Jaw: Case Report. Braz Dent J. 2016;27(3):353-8. PMID: 27224573; doi: 10.1590/01036440201600662.

13. Malan J, Ettinger K, Naumann E, Beirne OR. The relationship of denosumab pharmacology and osteonecrosis of the jaws. Oral Surg Oral Med Oral Pathol Oral Radiol. 2012;114(6):671-6. PMID: 23159111; doi: 10.1016/j.0000.2012.08.439.

14. Fusco V, Santini D, Armento G, Tonini G, Campisi G. Osteonecrosis of jaw beyond antiresorptive (bone-targeted) agents: new horizons in oncology. Expert Opin Drug Saf. 2016;15(7):925-35. PMID: 27074901; doi: 10.1080/14740338.2016.1177021.

15. Santos M de O. Estimativa 2018: incidência de câncer no Brasil. Revista Brasileira de Cancerologia. 2018; 64(1):119-20. doi: 10.32635/2176-9745. RBC.2018v64n1.115.

16. Martins MA, Martins MD, Lascala CA, et al. Association of laser phototherapy with PRP improves healing of bisphosphonaterelated osteonecrosis of the jaws in cancer patients: a preliminary study. Oral Oncol. 2012;48(1):79-84. PMID: 21940198; doi: 10.1016/j. oraloncology.2011.08.010.

17. Farias DS, Zen Filho EV, de Oliveira TF, et al. Clinical and image findings in bisphosphonate-related osteonecrosis of the jaws. J Craniofac Surg. 2013;24(4):1248-51. PMID: 23851781; doi: 10.1097/ SCS.0b013e3182902b91.

18. Rabelo GD, Assunção JN Jr, Chavassieux P, et al. Bisphosphonate-Related Osteonecrosis of the Jaws and Its Array of Manifestations. J Maxillofac Oral Surg. 2015;14(3):699-705. PMID: 26225065; doi: 10.1007/s12663014-0707-8.

19. Mathias Duarte LF, dos Reis HB, Tucci R, Dib LL. Bisphosphonate-related osteonecrosis of the jaws: analysis of a case series at a dental school. Spec Care Dentist. 2014;34(2):77-83. PMID: 23875734; doi: 10.1111/ scd. 12023

20. Heggendorn FL, Leite TC, Cunha KS, et al. Bisphosphonate-related osteonecrosis of the jaws: Report of a case using conservative protocol. Spec Care Dentist. 2016;36(1):43-7.PMID:26782365; doi: 10.1111/scd.12143.

21. Antonini F, Pereira CCS, Parente EV, Azambuja FG. Management of osteonecrosis of the jaws in patients with history of bisphosphonates therapy. J Craniofac Surg. 2010;21(6):1962-6. PMID: 21119470; doi : 10.1097/SCS.0b013e3181f4ee4e.

22. Maluf G, Caldas RJ, Silva Santos PS. Use of leukocyte-and platelet-rich fibrin in the treatment of medication-related osteonecrosis of the jaws. J Oral Maxillofac Surg. 2018;76(1):88-96. PMID: 28675810; doi: 10.1016/j. joms.2017.06.004.

23. Momesso GAC, de Souza Batista FR, de Sousa CA, et al. Successful use of lower-level laser therapy in the treatment of medication-related 
osteonecrosis of the jaw. J Lasers Med Sci. 2017;8(4):201-3. PMID: 29071028; doi: 10.22037/jlms.v8i4.16701.

24. de Oliveira Ruellas AM, Peruzzo DC, Napimoga MH. Managing bisphosphonate-related osteonecrosis of the jaws with xenografts: a case report. Clin Case Rep. 2017;5(8):1395-400. PMID: 28781866; doi: 10.1002/ccr3.1085.

25. Fernando de Almeida Barros Mourão C, Calasans-Maia MD, Del Fabbro M, et al. The use of Platelet-rich Fibrin in the management of medicationrelated osteonecrosis of the jaw: A case series. J Stomatol Oral Maxillofac Surg. 2020;121(1):84-9. PMID: 30794883; 10.1016/j.jormas.2019.02.011.

26. Santos M, Silveira K, Souza N, Costa D, Inaoka S. Extensive osteonecrosis of the maxilla caused by bisphosphonates: Report of a rare case. J Clin Exp Dent. 2019;11(2):e203-e207. PMID: 30805126; doi: 10.4317/ jced.55151.

27. Hoff AO, Toth BB, Altundag K, et al. Frequency and risk factors associated with osteonecrosis of the jaw in cancer patients treated with intravenous bisphosphonates. J Bone Miner Res. 2008;23(6):826-36. PMID: 18558816; doi: 10.1359/jbmr.080205.

28. Barasch A, Cunha-Cruz J, Curro F, et al. Dental risk factors for osteonecrosis of the jaws: a CONDOR case-control study. Clin Oral Investig. 2013;17(8):1839-45. PMID: 23212125; doi: 10.1007/s00784012-0880-4

29. O'Ryan FS, Lo JC. Bisphosphonate-related osteonecrosis of the jaw in patients with oral bisphosphonate exposure: clinical course and outcomes. J Oral Maxillofac Surg. 2012;70(8):1844-53. PMID: 22595135; doi: 10.1016/j.joms.2011.08.033

30. Mercer E, NortonT, Woo S, et al. Ninety-one osteoporosis patients affected with bisphosphonate-related osteonecrosis of the jaw: a case series. Calcif Tissue Int. 2013;93(3):241-8. PMID: 23756612; doi: 10.1007/s00223-0139747-1.

31. Di Fede O, Bedogni A, Giancola F, et al; BRONJ in patients with rheumatoid arthritis: a multicenter case series. Oral Dis. 2016;22(6):543-8. PMID: 27062502; doi: 10.1111/odi.12490.

32. Vahtsevanos K, Kyrgidis A, Verrou E, et al. Longitudinal cohort study of risk factors in cancer patients of bisphosphonate-related osteonecrosis of the jaw. J Clin Oncol. 2009;27(32):5356-62. PMID: 19805682; doi: 10.1200/JCO.2009.21.9584.

33. Saad F, Brown JE, Van Poznak C, et al. Incidence, risk factors, and outcomes of osteonecrosis of the jaw: integrated analysis from three blinded activecontrolled phase III trials in cancer patients with bone metastases. Ann Oncol. 2012;23(5):1341-7. PMID: 21986094; doi: 10.1093/annonc/mdr435.

34. Fehm T, BeckV, Banys M, et al. Bisphosphonate-induced osteonecrosis of the jaw (ONJ): Incidence and risk factors in patients with breast cancer and gynecological malignancies. Gynecol Oncol. 2009;112(3):605-9. PMID: 19136147; doi: 10.1016/j.ygyno.2008.11.029.

35. Pichardo SE, Kuijpers SC, van Merkesteyn JP. Bisphosphonaterelated osteonecrosis of the jaws: Cohort study of surgical treatment results in seventy-four stage II/III patients. J Craniomaxillofac Surg. 2016;44(9):1216-20. PMID: 27421170; doi: 10.1016/j.jcms.2016.06.016.
36. Blus C, Giannelli G, Szmukler-Moncler S, Orru G. Treatment of medicationrelated osteonecrosis of the jaws (MRONJ) with ultrasonic piezoelectric bone surgery. A case series of 20 treated sites. Oral Maxillofac Surg. 2017;21(1):41-8. PMID: 27924427; doi: 10.1007/s10006-016-0597-7.

37. Bagan JV, Cibrian RM, Lopez J, et al. Sclerosis in bisphosphonate-related osteonecrosis of the jaws and its correlation with the clinical stages: study of 43 cases. Br J Oral Maxillofac Surg. 2015;53(3):257-62. PMID: 25560326; doi: 10.1016/j.bjoms.2014.12.004

38. Nisi M, La Ferla F, Karapetsa D, et al. Conservative surgical management of patients with bisphosphonate-related osteonecrosis of the jaws: a series of 120 patients. Br J Oral Maxillofac Surg. 2016;54(8):930-5. PMID: 27418080; doi: 10.1016/j.bjoms.2016.06.015.

39. Sammut S, Malden N, Lopes V, Ralston S. Epidemiological study of alendronate-related osteonecrosis of the jaw in the southeast of Scotland. Br J Oral Maxillofac Surg. 2016;54:501-505.

40. Nomura T, Shibahara T, Uchiyama T, et al. Bisphosphonate-related osteonecrosis of jaw (BRONJ) in Japanese population: a case series of 13 patients at our clinic. Bull Tokyo Dent Coll. 2013;54(2):117-25. PMID: 23903583; doi: 10.2209/tdcpublication.54.117.

41. Kim HJ, Tae-Jun P, Ahn KM. Bisphosphonate-related osteonecrosis of the jaw in metastatic breast cancer patients: a review of 25 cases. Maxillofac Plast Reconstr Surg. 2016;38(1):6. PMID: 26870717; doi: 10.1186/s40902-016-0052-6.

42. Hong SO, Lee CY, Jung J, et al. A retrospective study of osteomyelitis and osteonecrosis of the jaws and its etiologic implication of bisphosphonate in Asians. Clin Oral Invest. 2017;21(5):1905-11. PMID: 27771829; doi: 10.1007/s00784-016-1973-2.

43. Kim TH, Seo WG, Koo CH, Lee JH. Evaluation of the predisposing factors and involved outcome of surgical treatment in bisphosphonate-related osteonecrosis of the jaw cases including bone biopsies. J Korean Assoc Oral Maxillofac Surg. 2016;42(4):193-204. PMID: 27595086; doi: 10.5125/ jkaoms.2016.42.4.193.

44. Guazzo R, Sbricoli L, Ricci S, et al. Medication-related osteonecrosis of the jaw and dental implants failures: a systematic review. J Oral Implantol. 2017;43(1):51-7. PMID: 28231038; doi: 10.1563/aaid-joi-16-00057.

45. Kyrgidis A, Vahtsevanos K, Koloutsos G, et al. Bisphosphonate-related osteonecrosis of the jaws: a case-control study of risk factors in breast cancer patients. J Clin Oncol. 2008;26(28):4634-8. PMID: 18574158; doi: 10.1200/JCO.2008.16.2768.

46. Watters AL, Hansen HJ, Williams T, et al. Intravenous bisphosphonaterelated osteonecrosis of the jaw: long-term follow-up of 109 patients. Oral Surg Oral Med Oral Pathol Oral Radiol. 2013;115(2):192-200. PMID: 23036797; doi: 10.1016/j.000o.2012.05.017.

47. Bodem JP, Schaal C, Kargus S, et al. Surgical management of bisphosphonate-related osteonecrosis of the jaw stages II and III. Oral Surg Oral Med Oral Pathol Oral Radiol. 2016;121(4):367-72. PMID: 26795450; doi: 10.1016/j.000o.2015.10.033.

48. Nørholt SE, Hartlev J. Surgical treatment of osteonecrosis of the jaw with the use of platelet-rich fibrin: a prospective study of 15 patients. 
Int J Oral Maxillofac Surg. 2016;45(10):1256-60. PMID: 27179556; doi: 10.1016/j.ijom.2016.04.010.

49. Kim SM, Eo MY, Kim YS, Lee SK. Histochemical observation of bony reversal lines in bisphosphonate-related osteonecrosis of the jaw. Oral Surg Oral Med Oral Pathol Oral Radiol. 2017;123(2):220-8. PMID: 27989705; doi: 10.1016/j.000o.2016.09.225.

50. Dimopoulos MA, Kastritis E, Anagnostopoulos A, et al. Osteonecrosis of the jaw in patients with multiple myeloma treated with bisphosphonates: evidence of increased risk after treatment with zoledronic acid. Haematologica. 2006;91 (7):968-71. PMID: 16757414.

51. Zervas K, Verrou E, Teleioudis Z, et al. Incidence, risk factors and management of osteonecrosis of the jaw in patients with multiple myeloma: a single-centre experience in 303 patients. $\mathrm{Br} J$ Haematol. 2006;134(6):620-3. PMID: 16889620; doi: 10.1111/j.13652141.2006.06230.x.

52. Pozzi S, Marcheselli R, Sacchi S, et al. Bisphosphonate-associated osteonecrosis of the jaw: a review of 35 cases and an evaluation of its frequency in multiple myeloma patients. Leuk Lymphoma. 2007;48(1):56-64. PMID: 17325848; doi: 10.1080/10428190600977690.

53. Karasneh JA, Al-Eryani K, Clark GT, Sedghizadeh PP. Modified protocol including topical minocycline in orabase to manage medication-related osteonecrosis of the jaw cases. J Oral Pathol Med. 2016;45(9):718-20. PMID: 26750149; doi: 10.1111/jop.12419.

Authors' contributions: Maciel AP: conception, design, acquisition, analysis and interpretation of this work, drafting the work and revising it critically for important intellectual content; Quispe RA: substantial contributions to the conception, design and acquisition of data for the work, drafting the work and revising it critically for important intellectual content; Martins LJO: substantial contributions to the conception or design and the acquisition of data for the work; Caldas RJ: substantial contributions to the design, analysis of the work and revising it critically for important intellectual content; and Santos PSS: final approval of the version to be published and agreement to be accountable for all aspects of the work, to ensure that questions relating to the accuracy or integrity of any part of the work are appropriately investigated and resolved. All authors approved the final version for publication

Sources of funding: This study was financed in part by the Coordenação de Aperfeiçoamento de Pessoal de Nível Superior (CAPES), Brazil, under finance code 001

Conflict of interest: The authors declare that there was no conflict of interest
Date of first submission: August 10, 2019

Last received: February 10, 2020

Accepted: May 15, 2020

\section{Address for correspondence:}

Aloizio Premoli Maciel

Departamento de Cirurgia, Estomatologia, Patologia e Radiologia Alameda Dr. Otávio Pinheiro Brisola, 9-75

Vila Nova Cidade Universitária — Bauru (SP) — Brasil

CEP 17012-901

Tel. (+55 14) 3235-800

Fax. (+55 14) 98800-1822

E-mail: aloiziopremoli@usp.br 